\title{
Efficient Energy Management Technique for Optimal Resource Allocation in a Wireless Powered Communication Network
}

\author{
Ude Valentine Chinonso \\ Department of Electrical and Electronic Engineering \\ Federal University of Technology, Owerri \\ Nigeria
}

\author{
Nosiri Onyebuchi Chikezie \\ Department of Electrical and Electronic Engineering \\ Federal University of Technology, Owerri \\ Nigeria
}

\begin{abstract}
The study focused on achieving optimal resource allocation for Wireless Powered Communication Network (WPCN) using efficient energy management scheme. The proposed WPCN model was developed, structured with two Radio frequency channels viz- the RF energy transfer channel and the data communication. Analytical expressions were deduced to obtain optimal transmission power and system efficiency. Energy Management Scheme was applied in the system design to minimize the amplifier power consumption in the power beacon and guarantees the power beacon in receiving sensor measurement reports from the sensor node as frequently as required by a sensor application. It ensures optimal consumption of the harvested energy for continuous operation by the sensor node. A testbed was developed to measure the parameters and the system model characteristics which facilitates adequate evaluation of the energy management scheme. The results obtained showed that the proposed scheme adaptively controls the WPCN for optimal energy neutrality and high-efficiency RF power transfer. The proposed energy management scheme was observed to consume less than $50 \%$ of the power consumed by the no amplifier duty cycling scheme.
\end{abstract}

Keywords- Wireless power Communication Networks, Wireless Sensor Networks, Energy Management, Harvest-thenTransmit,

\section{INTRODUCTION}

Sensor networks and Internet-of-Things (IoT) are envisioned to make life more convenient and productive by connecting devices to collect data, make intelligent decisions and perform autonomous controls without human interventions. One of the major concerns in developing these systems is the energy consumption, which is vital not only in the data processing but also in the end-devices sensing and data exchange. Traditionally, fixed energy sources such as batteries have been used as the major power source for energy-constrained wireless networks like the sensor networks, with the disadvantage of limiting the operation time of the network. It is expected that devices should be sustainable even without a battery or with a small battery and the lifetime of wireless networks increased by replacing or recharging the batteries. However, it may be inconvenient, expensive, and even hazardous (in toxic environments) or impractical (for sensors implanted in human bodies). Therefore in this context, wireless power transfer has received high attention as a way of charging the devices without battery replacement. Also as an alternative solution to these problems, recent discoveries and innovations have been developed. These include the system of energy harvesting which has gained a lot of attention by researchers, since it provides a more cost-effective and truly infinite supply of energy to wireless networks by tapping and harvesting energy from the Environment. Energy harvesting by wireless networks enables wireless devices to harvest energy from ambient and natural renewable sources, such as solar and wind source, providing a green and renewable energy supply for wireless communication systems. However, due to the intermittent nature of renewable energy sources, the energy collected at the receiver is not controllable, and the communication devices may not always be able to harvest sufficient energy. On the other hand, it has been shown that wireless receivers can also harvest energy from Radio Frequency (RF) signals, which is known as Wireless Energy Transfer (WET) [1]. Since the RF signals are generated by dedicated devices, this type of energy source is more stable than natural renewable sources.

Recent works generally considered the power splitting ratio at the receiver side to study the fundamental tradeoff between the achievable throughput and the harvested energy [1]. The second line of researches in WET pursues Wireless Powered Communication Networks (WPCNs), where the wireless devices are first powered by WET and then use the harvested energy to transmit data signals [1]. In WPCN the power source (batteries) of wireless communication devices can be replenished remotely by means of microwave wireless power transfer (WPT) technology. When compared to conventional battery powered networks, WPCN eliminates the need of manual battery replacement or recharging, which helps to effectively reduce the operational cost and enhance communication performance. Also, WPCN allows for full control over its power transfer, where the transmit power, waveforms, and occupied time/frequency dimensions are all tunable for providing a much more stable energy supply under different physical conditions and service requirements. This is in sharp contrast to Energy Harvesting (EH) based approaches, in which wireless devices opportunistically tap from renewable energy in an environment not dedicated to power the wireless devices such as solar power and ambient Radio Frequency (RF) transmission [2]. Due to the fact that the availability and strength of such renewable energy sources are mostly unpredictable and constantly varying with time, a more stable and on-demand energy supply to Wireless 
Devices WDs (WDs) is often not feasible with EH-based methods. Current WPT technology can effectively transfer tens of microwatts RF power to WDs from a distance of more than 10 meters, while there is still significant room for improving the magnitude and range with future advancement in WPT [3]. This makes WPCN potentially suitable for a broad range of low-power applications with operating power requirements of up to several milliwatts, such as wireless sensor networks (WSNs) and radio frequency identification (RFID) networks.

Despite the potential performance improvement brought by WPCN, building efficient WPCNs is a challenging problem in practice. Network system throughput has been improved and enhanced against the challenge of energy utilization efficiency in WPCN. Passive receivers (small sensor) nodes uplink data transmission is only possible after the receivers have harvested a sufficient amount of energy from the energy source in the downlink. Therefore energy efficiency of the network through joint time allocation and power control is a major challenge in these networks. The challenge of physical constraint on the energy usage motivated a "harvest-then-transmit" design. Management of time for energy harvesting in the downlink to a level that leads to a higher amount of harvested energy used in the uplink needs to be explored. This however, implies that there might be less time for uplink transmission resulting in a lower transmission data rate.

In order to meet the green communication requirement in future wireless networks, Energy Efficient (EE) WPCN design is of great importance to researchers and the industry alike. If the Energy Efficiency is maximized, the total power (or energy) consumption of the system will be significantly minimized for WPCNs perspective. For this reason, a joint data and energy transfer optimization framework is needed in addition to a user scheduling scheme to minimize the system energy consumption. Therefore, the idea of optimization of resources and energy consumption efficiency for WPCNs is very important. The recharging of batteries in hazardous and toxic environments or impractical locations such as sensors implanted in human bodies cannot be possible without wireless power transfer. This also has provided a means of charging devices without battery replacement. These WPTenabled wireless sensor networks (WSNs) and radio frequency identification (RFID) networks are currently reshaping the field of Internet-of-things (IoT) and machineto-machine (M2M) communications [4].

This work investigates how to optimally design a wireless powered communication network with efficient power requirements. To this end, an optimization algorithm is formulated to minimize the total energy consumption at the Remote Radio Units (RRUs) by jointly optimizing energy harvesting and time assignment, where the network energy consumption including basic circuit and information processing energy consumption at the WD is taken into account in order to achieve a more practical and general system design. To solve this non-convex optimization problem, an efficient solution method is presented on the basis of variable substitutions and timeframe - activity allocation technique. The optimality of the proposed solution method is analyzed in other to study resource allocation among the transmitting WDs, where the receiver carries out decoding and/or harvesting operations. Finally a comparison of the performance of the proposed energy management scheme and the no amplifier duty cycling scheme is performed.

\section{REVIEW OF RELATED WORKS ON RESOURCE ALLOCATION IN WCPNS}

Hybrid Access Point (HAP) aided WPCNs has wide applications and a lot of works have been done relative to its applications. For example, in [5], the sum-throughput of all users was maximized via time assignment for uplink and downlink, where however, both users and the HAP were equipped with single antenna. As is known, with beamforming technology, both information and energy transmission efficiency of the multi-antenna HAP can be greatly enhanced. Also, some other works investigated the multi antenna HAPaided WPCNs such as in [6], the authors studied the throughput maximization problem via optimizing energy beamforming. Meanwhile, in order to meet the green communication requirement in further wireless networks, Energy Efficient (EE) WPCN design is of great importance as was discussed in [7]. However, in [8] and [9], the Energy Efficiency was maximized, this was done by developing a cooperative sharing model for cognitive wireless powered communication networks (CWPCN), which leverages the notion of cooperation between primary and secondary systems in cognitive radio (CR) networks to accomplish primary transmission early and vacate opportunity for secondary transmission. With the proposed spectrum sharing model, they investigated the optimal design for such a cooperative CWPCN with both amplify-and-forward (AF) and decodeand-forward (DF) protocols to maximize its uplink energy efficiency. The energy efficiency optimization problem was decomposed into two sub-problems for uplink scheduling and cooperative power control. They also proposed a suboptimal solution to tackle the unavailable information on channel condition between the primary transmitter and secondary users. Numerical results showed that the DF protocol outperforms the AF protocol in general and the suboptimal solution was slightly outperformed by the optimal one.

In [4] and [10], the total power (or energy) consumption of the system was minimized for WPCNs perspective. The energy efficiency (EE) in multiple relay-aided OFDM systems was investigated, where decode-and-forward (DF) relay beamforming was introduced to enable the information transmission. To explore the system performance behavior with user fairness for such a system, an optimization problem was formulated to maximize the EE by jointly considering multiple factors, i.e., the transmission mode selection (DF relay beamforming or direct-link transmission), the helping relay set selection, the subcarrier assignment and the power allocation at the source and relays on subcarriers, under nonlinear proportional rate fairness constraints, where both transmit power consumption and linearly rate- 
dependent circuit power consumption were taken into account. To solve the non-convex optimization problem, they proposed a low-complexity scheme to approximate it. The effects of the circuit power consumption on system performance was also studied and it was observed that with either the constant or the linearly rate-dependent circuit power consumption, system, EE grows with the increment of system average Channel-to Noise Ratio (CNR), but the growth rates showed different behaviors. For the constant circuit power consumption, system EE increasing rate was an increasing function of the average CNR, while for the linearly rate-dependent one, system EE increasing rate was a decreasing function of the average CNR. This observation was very important which indicated that by deducing the circuit dynamic power consumption per unit data rate, system EE could be greatly enhanced.

A joint data and energy transfer optimization framework was proposed in [10], where the focus was on radio frequency (RF) energy harvesting for multiuser multicarrier mobile wireless networks. In the framework, the mobile users were capable of harvesting energy from the dedicated subcarriers over which they communicated with the base station in addition to harvesting ambient RF signals. A joint data and energy transfer optimization framework for powering mobile wireless devices through RF energy harvesting while minimizing the overall power consumption was proposed. The proposed framework determines the optimal power resources that need to be allocated to meet data rate requirements of downlink and uplink communications. Simulations showed that substantial power savings were achieved by allowing the ambient RF energy harvesting as well as by exploiting the different system parameters.

In [4], a user scheduling scheme was presented to minimize the system energy consumption. A theoretical analysis on the energy consumption of the mobile terminals within the cloud was presented. User scheduling schemes were introduced in order to investigate when and how many users should participate in receiving from the Base Station in order to improve energy efficiency. Sub-channel and power allocation schemes were proposed with the objective to minimize the energy consumption of the considered system. Simulation results demonstrated the energy saving benefits of a mobile cloud and also illustrated the advantages of advocating wireless power transfer for the mobile cloud.

In [11], considered the so called harvest-then-transmit WPCNs, which typically utilize time division multiple access (TDMA) whose frame is divided into $\mathrm{N}+1$ subintervals; one for downlink energy transmission by the BS (i.e., an energy harvesting (EH) phase) and $\mathrm{N}$ successive uplink information transmissions (IT) by the EHUs. In a fading channel, the durations of the subintervals may be dynamically adjusted in order to maximize the sum-rate over the uplink of the WPCN [12]. However, such resource allocation proposed by is unfair to the cell-edge EHUs, yielding to the doubly near-far effect. This effect is caused by the distance-dependent large scale fading (i.e., the path loss), such that an EHU at further distance from the BS receives much less wireless energy than a nearer EHU, but also this far EHU has to transmit at more power for achieving the same rate as the nearer EHU. Hence, the differences in the achievable rates between the near and far EHUs are substantially higher than in the conventional (nonEH) cellular networks, which yields considerable system unfairness.

\section{WIRELESS POWERED COMMUNICATION NETWORK MODEL DEVELOPMENT}

The system model of the proposed WPCN is represented in Fig. 1. The WPCN made up of a power beacon and a sensor node. The fundamental role of the power beacon is to supply energy wirelessly to the sensor node via the RF energy transfer scheme. The sensor node depends majorly on the energy supplied wirelessly by the power beacon. The sensor measurement results are collected by the power beacon from the sensor node and controls the operation of the sensor node through a data communication link.

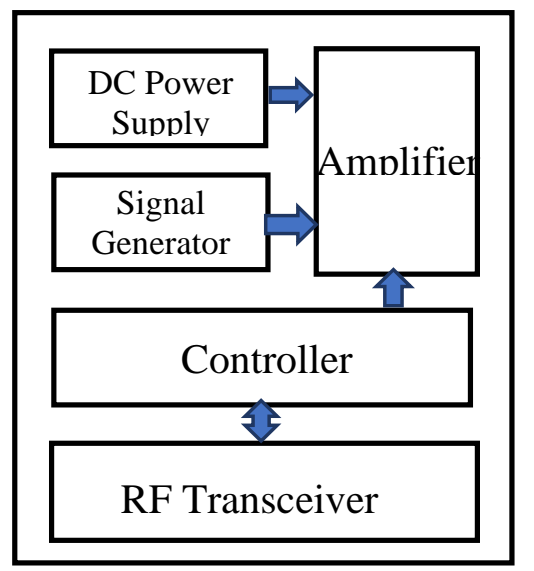

Power Beacon

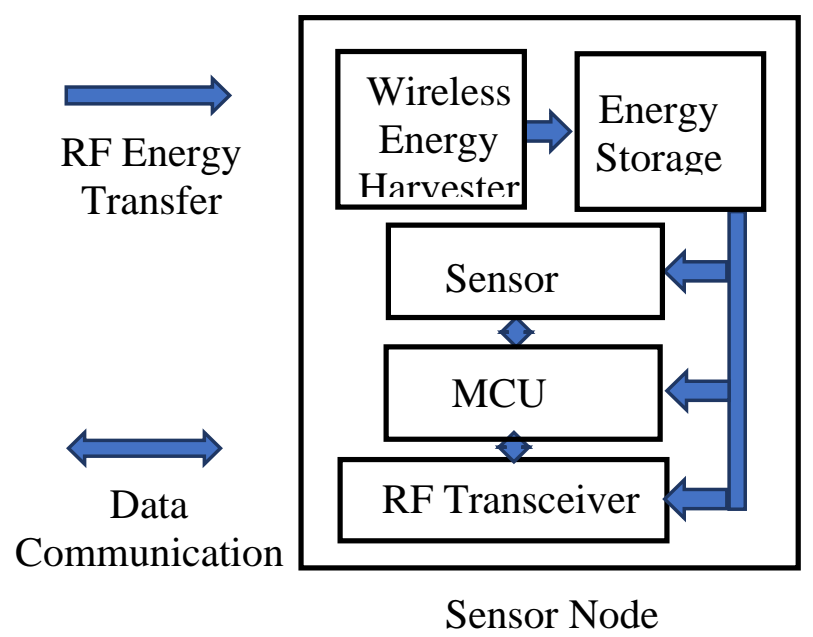

Figure 1 Block diagram of the Proposed WPCN model 
The signal generator generates a weak Continuous Wave (CW) RF source signal and feeds the RF source signal to the amplifier. The weak RF source signal from the signal generator is amplified by the amplifier and sends a highpower RF signal through the transmit antenna. The controller of the power beacon performs a power management function to ensure the stored energy of the sensor node has a sufficient energy level while minimizing power consumption of the amplifier. The control decision made by the controller is based on the sensor measurement report from the sensor node, which is delivered by the RF transceiver. At the sensor node, the wireless energy harvester receives the RF signal from the power beacon through the receive antenna. The wireless energy harvester performs RF-to-DC conversion by using a special rectifier circuit, the rectifier is embedded in the wireless energy harvester block. The DC power from the wireless energy harvester is stored in the energy storage. A Lithium Polymer (LiPo) battery was adopted in this project as the energy storage rather than a rechargeable battery. A Lithium polymer battery energy storage device was considered suitable for energy harvesting sensor nodes owing to the fact that it can tolerate rapid charging and discharging cycles. The entire sensor nodes is controlled by micro controller unit (MCU). The MCU extracts the sensing information from the sensors and transmits the report obtained to the power beacon through the RF transceiver. The WPCN system model uses two RF channels: the RF energy transfer channel and the data communication channel. These two channels uses different frequency bands of $920 \mathrm{MHz}$ and $2.4 \mathrm{GHz}$ respectively, and therefore there could be no interference between these channels.

\section{ENERGY MANAGEMENT SCHEME}

Generally, Energy management scheme is primarily designed to achieve the following: (a) to minimize the amplifier power consumption in the power beacon. (b) Ensures sensor measurement report is received by the power beacon as often as required by the sensor application. (c) Ensures a maximum stored energy $\mathrm{E}$ over the minimum stored energy $E_{\min }$ is actualized as a requirement for optimal sensor node operation and (d) guarantees optimal consumption of the harvested energy for continuous operation by the sensor node. Due to the inherent incoherence and conflicting challenges that exists amongst the four basic functions of the energy management scheme, a careful design approach was considered to ensure optimal balancing of the scheme. From the research study, the system was designed in a way that both the power beacon and the sensor node perform duty cycling. Figure 2 illustrates the timing diagram of the energy management scheme. The basic time unit for all operations of the energy management scheme is a frame, whose length is denoted by $\mathrm{T}_{\text {frame }}$. Each frame is indexed by $f$. The power beacon performs duty cycling on a frame-by-frame basis. During each frame, the power beacon turns $\mathrm{ON}$ the amplifier for $\mathrm{T}_{\mathrm{ET}}$ and turns $\mathrm{OFF}$ the amplifier for the rest of the frame. Then, the amplifier duty cycle, denoted by $\alpha$, is given by:

$$
\alpha=T_{E T} / T_{\text {frame }}
$$

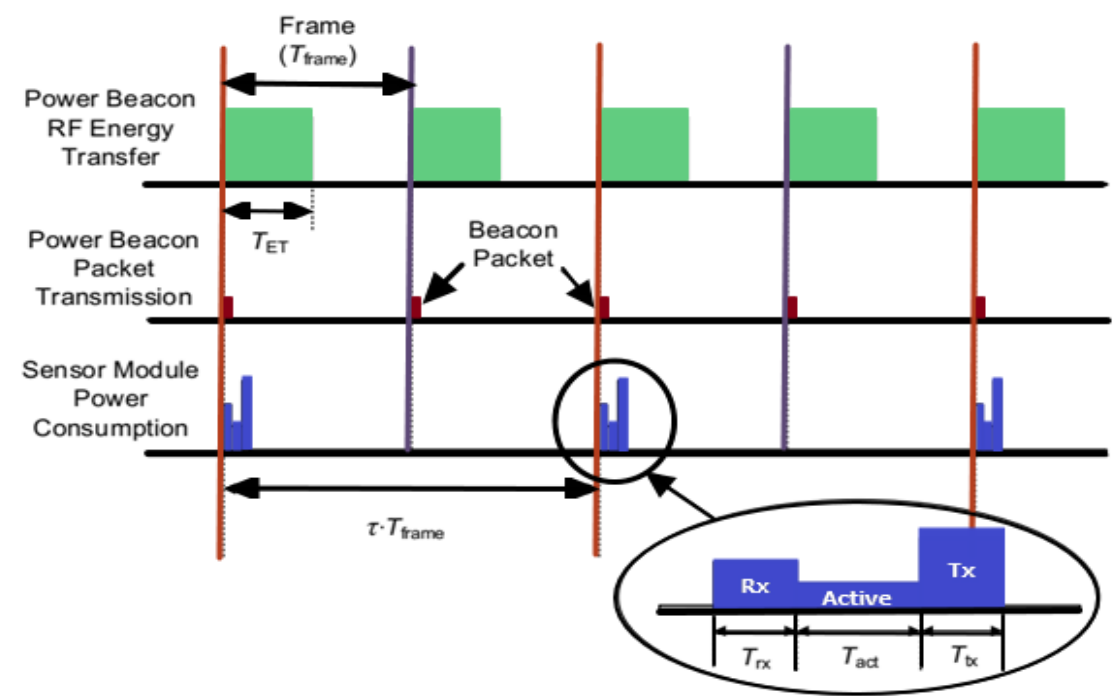

Figure 2. Timing diagram of the Designed Energy Management Scheme

When the amplifier is turned ON, the transmit power is set to $p_{t x}=Y$. Let $Y$ represent the energy transfer power. The energy transfer power satisfies $0<Y \leq Y_{\max }$ where $Y_{\max }$ denotes the maximum energy transfer power. The amplifier power consumption is given by

$$
p_{\text {cons }}=\boldsymbol{Y}=\boldsymbol{\theta}(\boldsymbol{Y})
$$

When the amplifier is turned off, the transmit power and the amplifier power consumption are both zero. Let $\alpha(f)$ and $\mathrm{T}(f)$ denote the amplifier duty cycle and the energy transfer power in frame $f$ respectively. The sensor node performs duty 
cycling that periodically wakes up the sensor node for a short time and then puts the sensor node into the idle mode to minimize the sensor module power consumption. A frame is a basic time unit of the duty cycling of the sensor node. For each frame, the sensor node can be in the awake state or in the sleep state. If the sensor node is in the awake state during a frame, the sensor node goes through the receive, active, and transmit modes in sequence for $T_{\mathrm{rx}}, T_{\mathrm{act}}$, and $T_{\mathrm{tx}}$, respectively, and it goes into the idle mode for the rest of the frame during $T_{\text {idle }}=T_{\text {frame }}-T_{\text {rx }}-T_{\text {act }}-T_{\text {tx }}$, as shown in Figure 2. Conversely, if the sensor node is in the sleep state during a frame, it stays in the idle mode for the entire frame. Let $a(f)$ denote an awake indicator that is 1 if the sensor node is in the awake state in frame $f$; and is 0 , otherwise. At the start of each frame, the power beacon sends a beacon packet that contains a control command to the sensor node. When the sensor node wakes up at the start of a frame, the sensor node is in the receive mode for time duration $T_{\mathrm{rx}}$ to receive a beacon packet from the power beacon. The sensor node wakes up slightly before the start of a frame to make sure the sensor node safely receives a beacon packet. After successful reception of a beacon packet, the sensor node turns off the RF transceiver and the mode of the sensor node is changed to the active mode. In the active mode, the sensor node performs sensor measurements and computation for time duration $T_{\text {act. }}$. The sensor measurements include the receive power, the stored energy, and other sensor measurements (e.g., temperature). During the time that the sensor node measures the receive power, the amplifier in the power beacon should be turned ON. Hence the energy management scheme makes the amplifier duty cycle larger than a predefined minimum amplifier duty cycle $\alpha_{\min }$ so that the amplifier is turned ON while the sensor node is in the active mode. The receive power measurement in frame $f$ is denoted by $S(f)$. In addition, the sensor node measures the sensor node voltage and calculates the stored energy. The stored energy measurement in frame $f$ is denoted by $E(f)$.

The sensor node prepares the sensor measurement report that includes $S(f), E(f)$, and other sensor measurements. The sensor node then sends a data packet containing the sensor measurement report in the transmit mode for time duration $T_{\mathrm{tx}}$. After the packet transmission is over, the sensor node is put into the idle mode. The sensor node wakes up again after the wake-up interval. The energy management scheme has three control parameters: the amplifier duty cycle, the energy transfer power, and the wake-up interval. The energy management scheme can dynamically decide the amplifier duty cycle, the energy transfer power, and the wake-up interval for each frame in which the sensor node is in the awake state. This decision is based on the receive power measurement $S(f)$ and the stored energy measurement $E(f)$ included in the sensor measurement report from the sensor node. The controller sends the decided wake-up interval to the sensor node by enclosing it in the control command in the beacon packet, and the sensor node adjusts the wake-up interval according to the received control command.

\section{WCPN SIMULATION USING TRUE TIME SIMULATOR TOOL.}

True Time (TT) is a MATLAB/Simulink based cosimulation tool. It comprises a kernel block that executes code and models, e.g., I/O tasks, control algorithms, and network interface drivers. The model, as implemented in the MATLAB/ Simulink environment using the True Time library, is shown in Figure 3.
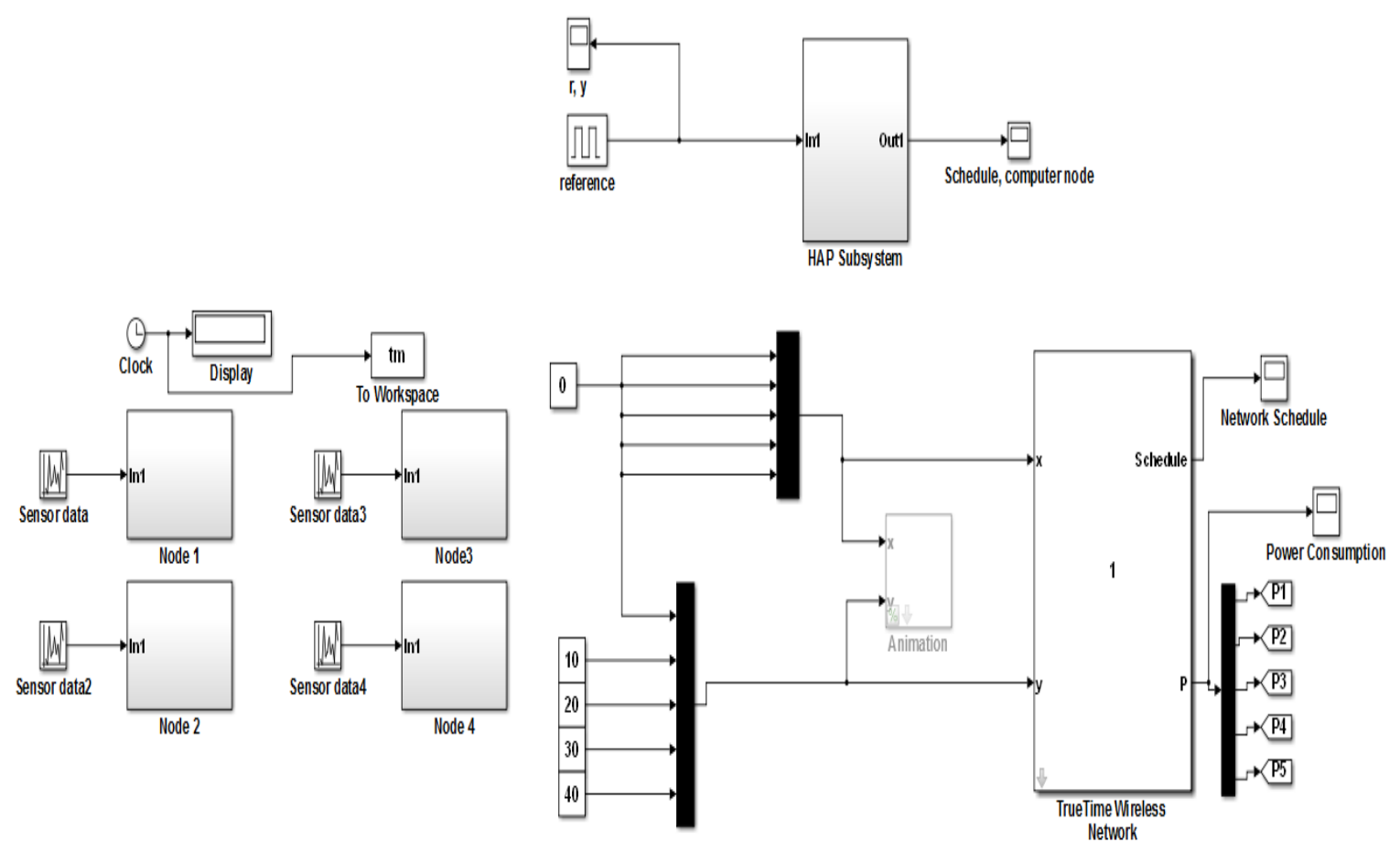

Figure 3. True Time block diagram of the System model 


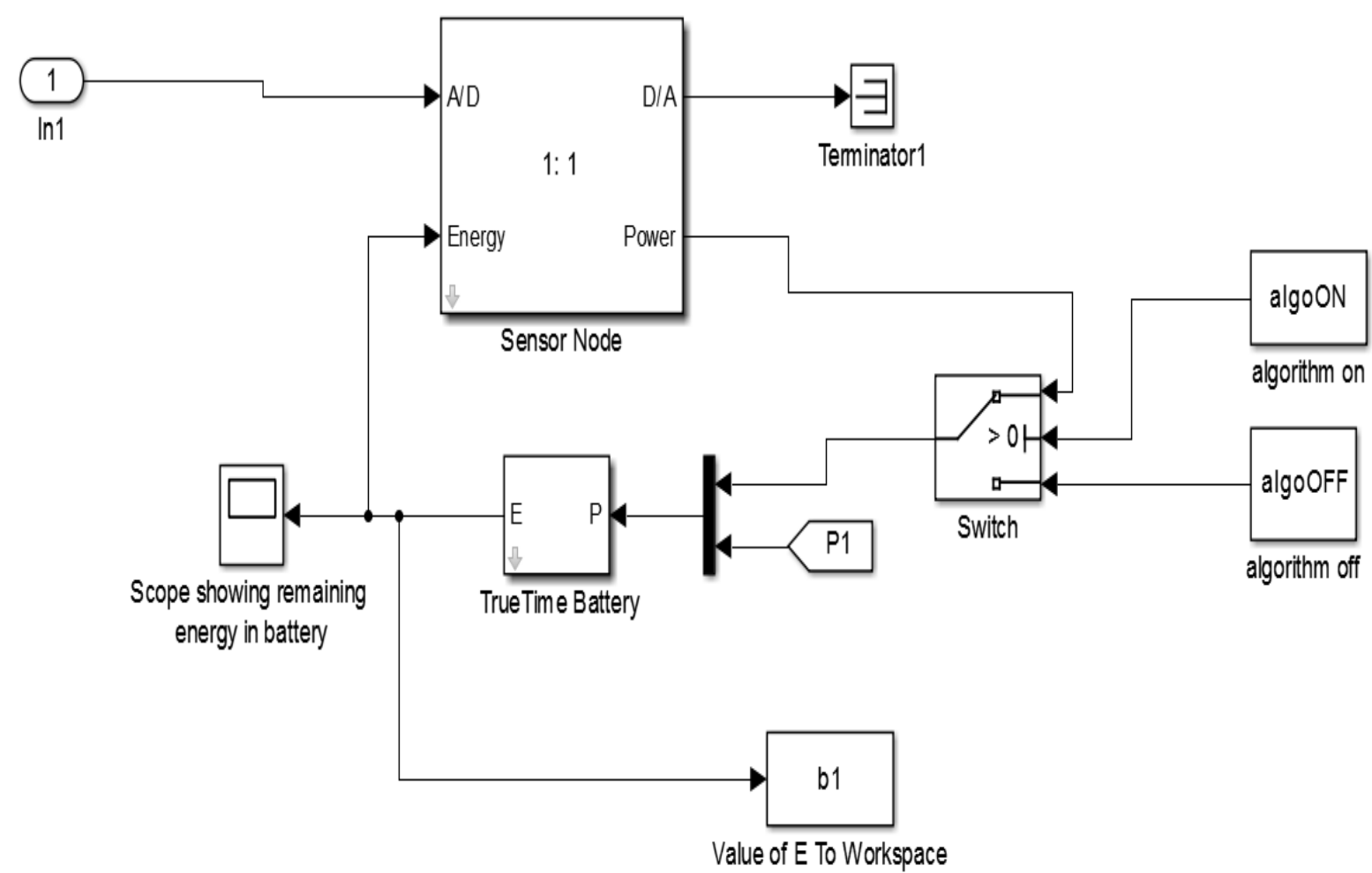

Figure 4: Internal Structure of Simulated Nodes.

Figure 3 shows the simulation environment for the WPCN model consisting of one HAP and four sensor nodes using Simulink and True Time simulator environment. The sensor nodes are fed with some random data signifying the quantity it is to measure. The wireless network block represents protocols and algorithms implemented for the overall network.

The internal structure of each node is shown in figure 4. Where each sensor node has an Analog to digital converter which converts the measured inputs into an 8-bit binary value to be processed by the microcontroller's CPU. It also has a digital to analog converter which is not used in the simulation, and therefore it is connected to the terminator block. The Energy block outputs the remaining energy in the respective node's battery while the Power block input determines the power consumption of the node. The switch block allows the user to simulate with or without the developed algorithm in order to see the different behaviors of the network in the respective modes. The simulation is initiated with the run button and lasts for approximately 10 seconds.

\section{RESULTS}

The experimental results on the WPCN testbed are presented to show the performance of the proposed resource allocation and management scheme. Simulation results were also obtained supporting the proposed WPCN system efficiency.
The length of a frame is $T_{\text {frame }}=100 \mathrm{~ms}$, the maximum energy transfer power is $\mathrm{T}_{\max }=2.3 \mathrm{~W}$, and the maximum sensor node voltage is $3.0 \mathrm{~V}$. In this paper, the power attenuation on the RF energy transfer channel was given in a $\mathrm{dB}$ scale. Table 1 shows the summary of results of the power amplifier energy consumption against the average transmit power. Figures 4-8 demonstrate the graphical representation of the system output relative to the amplifier power consumption levels.

TABLE 1. AVERAGE AMPLIFIER POWER CONSUMPTION AND TRASMITTED POWER AT DIFFERENT INPUTS.

\begin{tabular}{|l|r|r|r|}
\hline AAPC & $\begin{array}{l}\text { ATP } @ \boldsymbol{\alpha}=\mathbf{1}, \\
\mathbf{Y = 0 - 2 . 3 W}\end{array}$ & $\begin{array}{l}\text { ATP } \boldsymbol{a} \boldsymbol{\alpha}=\mathbf{0 - 1}, \\
\mathbf{Y}=\mathbf{0 . 5 W}\end{array}$ & $\begin{array}{l}\text { ATP } @ \boldsymbol{\alpha}=\mathbf{0 - 1}, \\
\mathbf{Y}=\mathbf{1 W}\end{array}$ \\
\hline 0.8084 & 0.7572 & 0.4759 & 0.5408 \\
\hline 1.9202 & 1.5094 & 1.0411 & 1.6472 \\
\hline 2.981 & 2.5157 & 1.3564 & 2.5965 \\
\hline 3.9916 & 3.3056 & 1.9108 & 3.3333 \\
\hline 5.1044 & 4.0208 & 2.653 & 3.7736 \\
\hline 6.8727 & 5.0941 & 4.1369 & 5.283 \\
\hline 8.0361 & 5.7233 & 5.1816 & 5.7848 \\
\hline 9.4018 & 6.5119 & 6.2893 & 6.7265 \\
\hline 11.3725 & 7.6731 & 7.4379 & 7.8616 \\
\hline 13.7994 & 9.2453 & 9.2727 & 9.2589 \\
\hline 15.7202 & 10.5031 & 10.4393 & 10.2559 \\
\hline 18.1978 & 11.9702 & 11.8868 & 12.1075 \\
\hline 19.7651 & 12.7673 & 12.6518 & 12.6162 \\
\hline
\end{tabular}



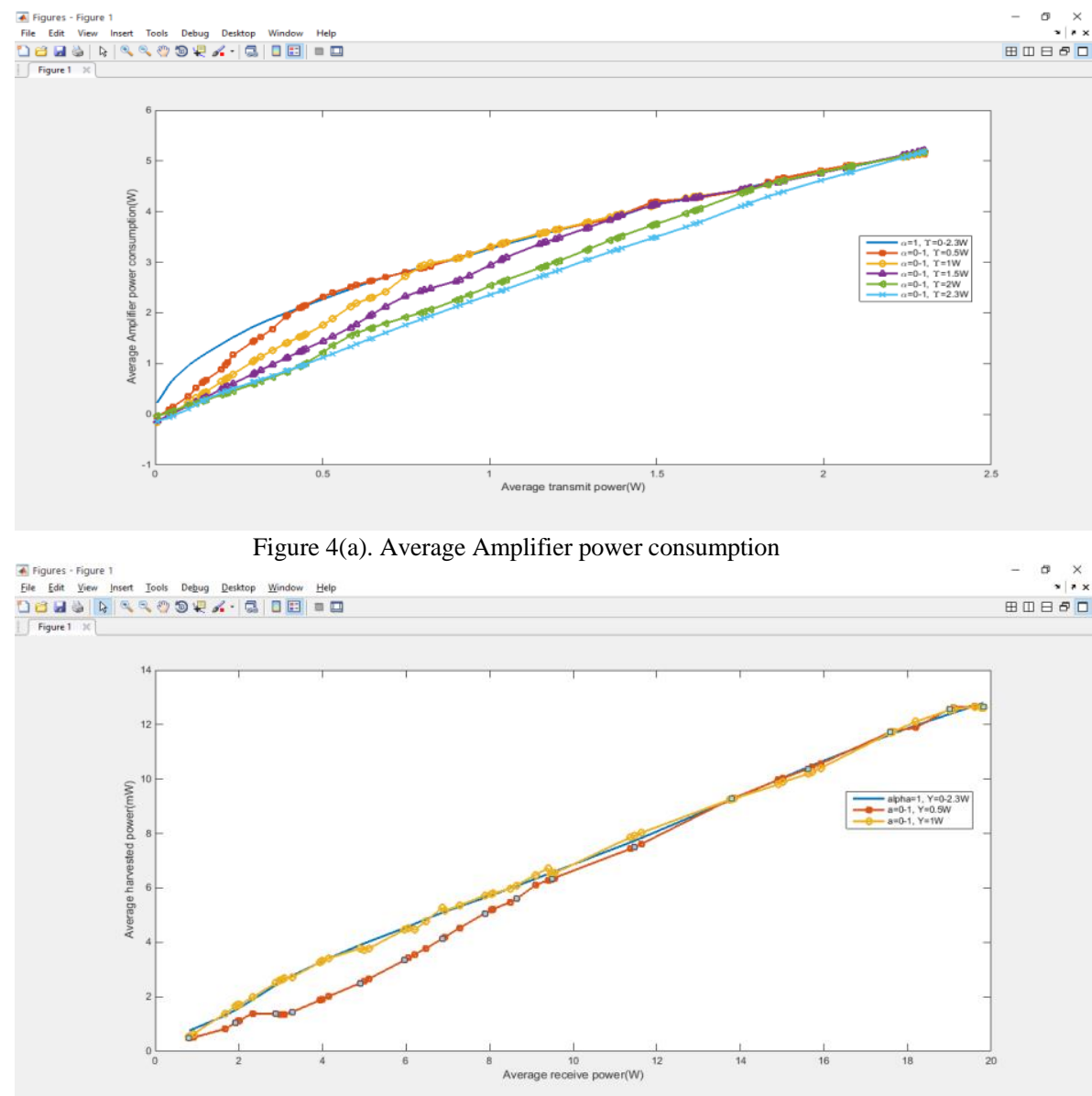

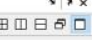

Figure 4(b). Average Harvested power

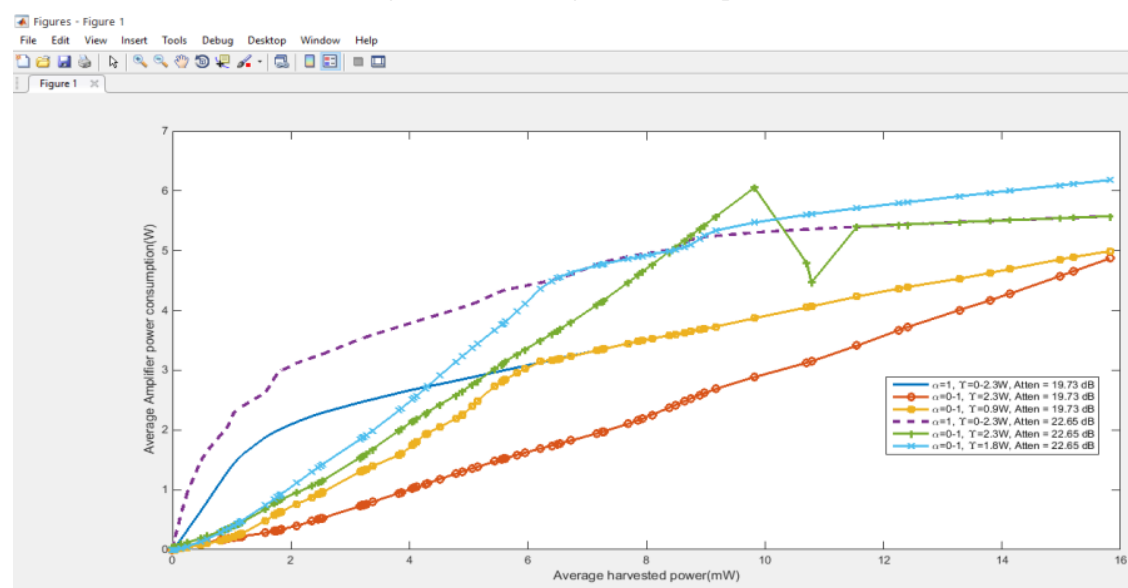

Figure 5 Average consumption of the power amplifier relative to average harvested power when amplifier duty cycling was incorporated. 
A) Figure - Figure 1

File Edt View Inset Tools Debug Deshtop Window Help

$-\underset{x \rightarrow \infty}{x} \times$

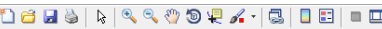

田田㫛

I] Figure $1 \times$

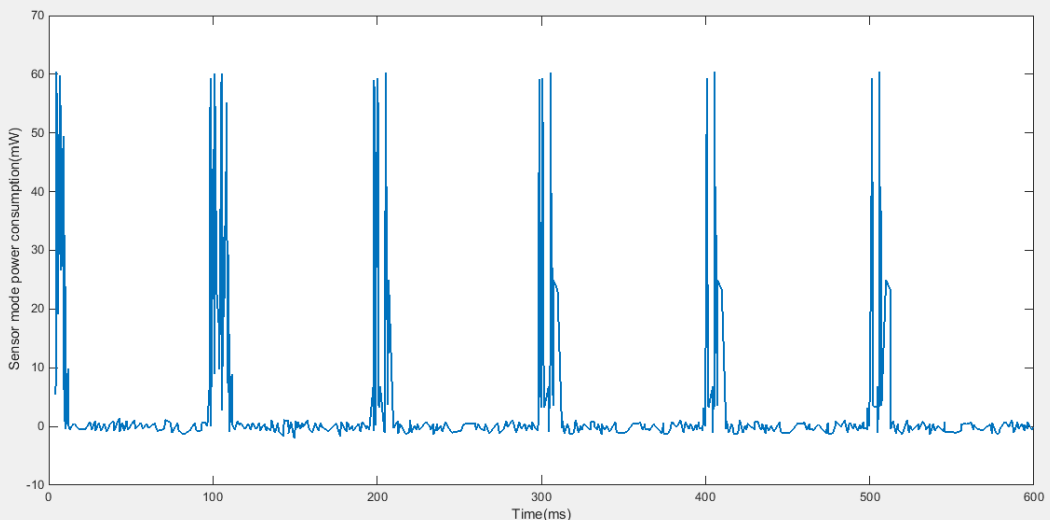

Figure 6(a) Power consumption in sensor mode over time (long time period)

Aigures - Figure
File Edit View

File Edit View Insert Tools Debug Desktiop Window Help

$-\operatorname{ar} x$

$\sqrt{\text { Figure } 1}$

田的口

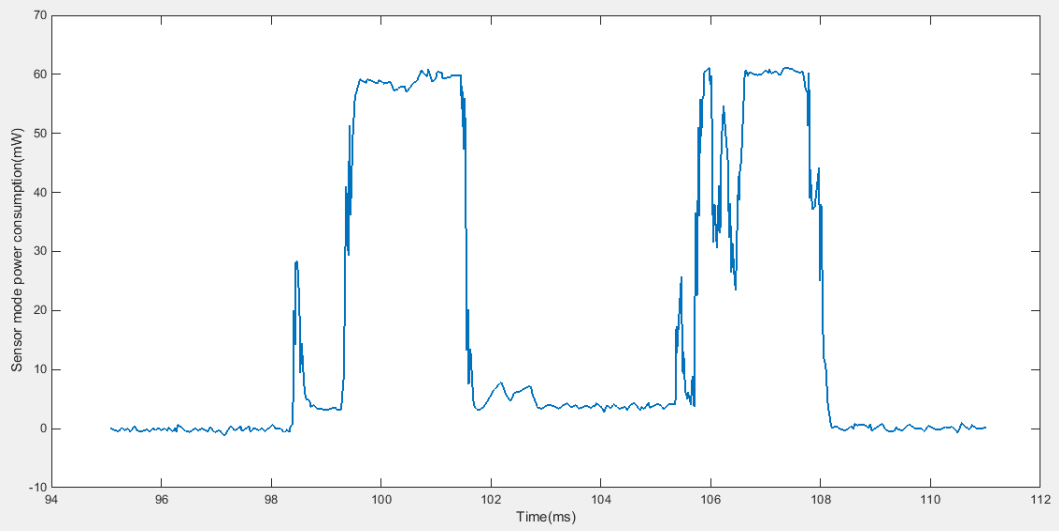

Figure 6(b) Power consumption in sensor mode over time (short time period)

(4) Figures - Figure

Dols Debug Deskiop Window Help

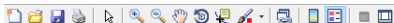

I) Figure 1

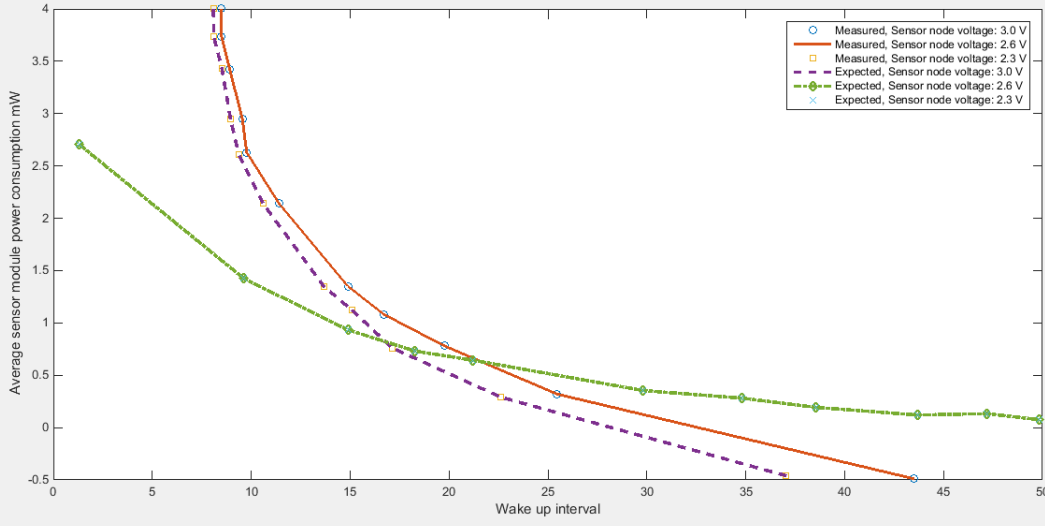

Figure 7. Average sensor module power consumption according to wake-up interval 


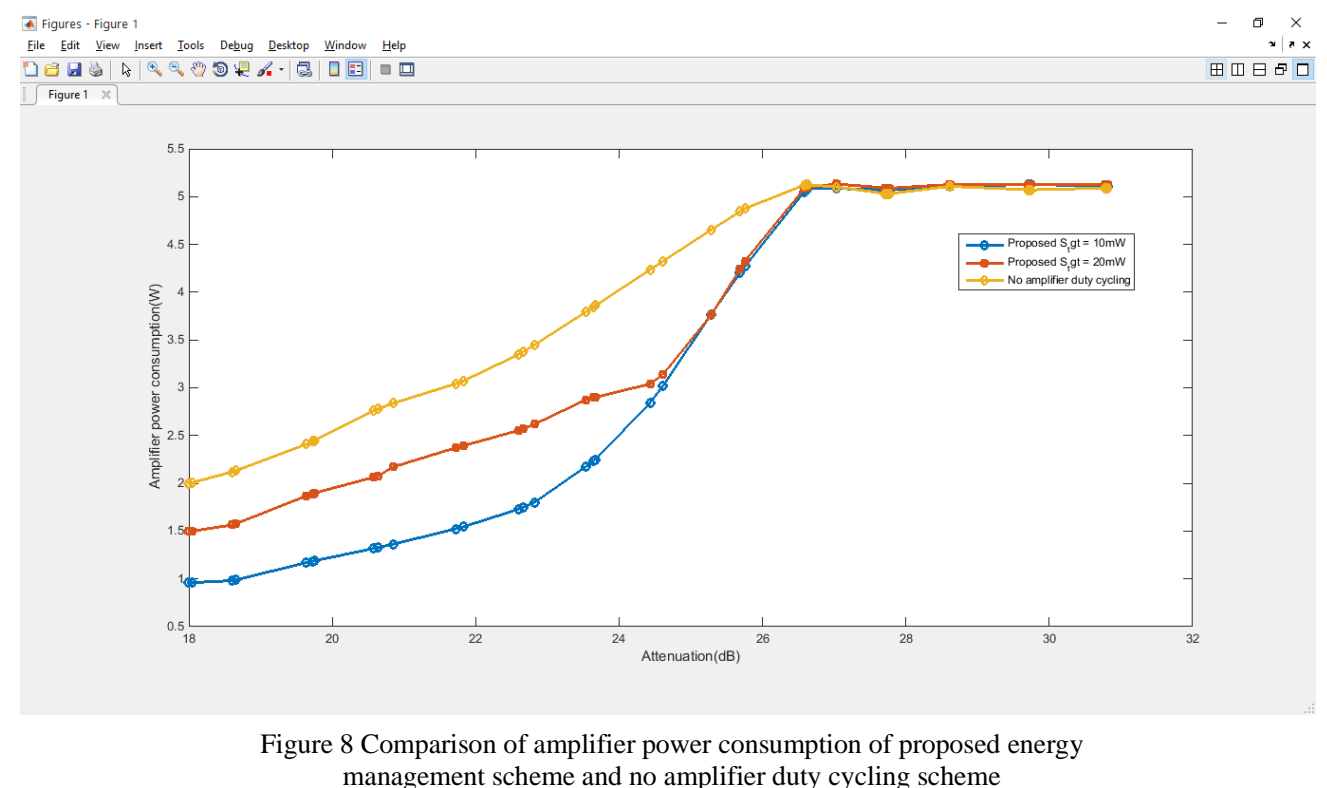

\section{DISCUSSION}

In Figures 4 and 5, the efficiency of the RF energy transfer when the amplifier duty cycling was applied is shown. Figure. 4(a) shows the average amplifier power consumption (i.e., $\Omega(\alpha, \mathrm{T})=\alpha \cdot(\mathrm{T}=\theta(\mathrm{T})))$ on $\mathrm{y}$-axis and the average transmit power (i.e., $\alpha \mathrm{T}$ ) on $\mathrm{x}$-axis, according to the amplifier duty cycle $\alpha$ and the energy transfer power T.

In Figure 5, it can be observed that lower average amplifier power consumption was achieved for a given average transmit power when the amplifier duty cycling was used with fixed $\mathrm{T}$, which is because of the characteristics of the PAE. Just as in Figure. 4(b), the average harvested power (i.e., $\alpha \cdot \eta E(E, h \mathrm{~T}) \cdot h \mathrm{~T})$ is shown on $\mathrm{y}$ axis and the average received power (i.e., $\alpha \cdot h \mathrm{~T}$ ) on the $\mathrm{x}$-axis respectively, according to $\alpha$ and the receive power (i.e., $h \mathrm{~T}$ ).

Since the wireless energy harvesting efficiency is maximized around $10 \mathrm{~mW}$, a higher average harvested power was obtained when received power is fixed to $10 \mathrm{~mW}$. Figure 5 also shows the average amplifier power consumption for achieving a given average harvested power, which is affected by both the PAE and the wireless energy harvesting efficiency, when the power attenuations are $19.73 \mathrm{~dB}$ and $22.65 \mathrm{~dB}$. It clearly shows the advantage of the amplifier duty cycling.

Figure 6 shows the sensor module power consumption overtime when the wake-up interval was set to $\tau=1$ and the sensor node voltage is 3.0 V. In Figure 6(a), it can be seen that the sensor module wakes up every $100 \mathrm{~ms}$ similarly to the timing diagram in Figure 6(b). From Figure 6(b), the time duration of each mode such that $T_{\mathrm{rx}}=2.34 \mathrm{~ms}, T_{\text {act }}=5.01 \mathrm{~ms}$, and $T_{\mathrm{tx}}=1.81 \mathrm{~ms}$ was derived.
Figure 7, shows the average sensor module power consumption according to the wakeup interval. In Figure 7, 'Expected' refers to the average sensor module power consumption obtained based on $T_{\mathrm{rx}}, T_{\mathrm{act}}, T_{\mathrm{tx}}$. In addition, the actual sensor node power consumption was measured by measuring current going through the sensor module. It can be seen that the expected sensor module power consumption well matches with the measured one.

Figure 8 shows the amplifier power consumption of the proposed energy management scheme and the no amplifier duty cycling scheme. From Figure 8 , it could be observed that the proposed scheme outperforms the no amplifier duty cycling scheme. When $S$ tgt $=20 \mathrm{~mW}$ and the power attenuation is less than $23 \mathrm{~dB}$, the proposed scheme only consumes less than half of the power consumed by the no amplifier duty cycling scheme.

\section{CONCLUSION}

A study on resource allocation algorithms for RF-based EH wireless communication networks, which are of interest were carried out. A parametric non-linear EH model was discussed, which facilitated the resource allocation algorithm design to enable efficient wireless powered communication networks. The algorithm designs were formulated as a nonconvex optimization problem for maximizing the throughput in WPCN systems. The problem formulations took into account the imperfectness of the transmission system and the non-linearity of the EH circuits in order to ensure robust resource allocation. The proposed resource allocation design optimization problems were optimally solved by advanced signal processing techniques using MATLAB and True Time simulator with simulink. Numerical results showing the potential gains in harvested power that was enabled by the proposed optimization wireless powered communication networks. 
Based on the stored energy evolution model, an energy management scheme was proposed for the energy neutral operation of the sensor node. The experimental results demonstrated that the proposed scheme adaptively controls the WPCN to achieve energy neutrality and high-efficiency RF power transfer. The proposed energy management scheme was confirmed to consume less than half of the power consumed by the no amplifier duty cycling scheme.

The research study considered the following recommendations:

1. This study was conceived for development of an improved energy management scheme for WPCN, it is highly recommended for implementation by industrial players and government agencies such as communication industries, Airports and the military for charging of surveillance systems and sensor networks.

2. This research study deployed only one HAP (Energy Beacon). Further studies should incorporate more than one HAP, which could enhance long range energy coverage.

3. Experimental investigation of the influence of different parameters such as attenuation and fading and its effect on resource management and QOS was not incorporated in the study due to equipment limitations and time constraints. It is suggested to be conducted in further studies.

4. Models for predicting the amount of energy required for WPCNs as a way of improving the current scheme, for example using artificial intelligence Technique's such as fuzzy logics and neural networks may also be carried out.

\section{REFERENCES}

[1] Q. Wu, M. Tao, D.W. Kwan, W. Chen, \& R. Schober, "EnergyEfficient Resource Allocation for Wireless Powered Communication Networks". IEEE Transactions on Wireless Communications, 15(3), 2312-2327. https://doi.org/10.1109/TWC.2015.2502590, 2016.

[2] M. Ku, W. Li, Y. Chen, \& K.J.R. Liu, "Advances in Energy Harvesting Communications: Past, Present, and Future Challenges", 1-23. 2014.

[3] S.B. Zhang, "Placement Optimization of Energy and Information Access Points in Wireless Powered Communication Networks". IEEE Trans. Wireless Commun, 2016

[4] Z. Chang, J. Gong, Y. Li, Z. Zhou, T. Ristaniemi, G. Shi, Z. Niu, "Energy Efficient Resource Allocation for Wireless Power Transfer Enabled Collaborative Mobile Clouds". IEEE Journal on Selected Areas in Communications, 34(12), 3438-3450. https://doi.org/10.1109/JSAC.2016.2611843, 2016.

[5] R. Zhang, "Throughput maximization in wireless powered communication networks with energy saving". IEEE Transactions on Wireless Communications, 2015-April(1), 516-520. https://doi.org/10.1109/ACSSC.2014.7094498, 2015

[6] D. Hwang, D.I. Kim, \& T.J. Lee, "Throughput Maximization for Multiuser MIMO Wireless Powered Communication Networks". IEEE Transactions on Vehicular Technology, 65(7), 5743-5748. https://doi.org/10.1109/TVT.2015.2453206, 2016

[7] K. Xiong,P. Fan, Y. Lu, \& K. Letaief, "Energy efficiency with proportional rate fairness in multirelay OFDM Networks". IEEE Journal on Selected Areas in Communications, 34(5), 1431-1447. https://doi.org/10.1109/JSAC.2016.2545479, 2016

[8] X. Lin, L. Huang, C. Guo,P. Zhang, M. Huang, \& J. Zhang, "Energy-Efficient Resource Allocation in TDMS Based Wireless Powered Communication Networks". IEEE Communications Letters, 7798(c), 1-1. https://doi.org/10.1109/LCOMM.2016.2639484, 2016

[9] S. Yin, Z. Qu, Z. Wang, \& L. Li, " Energy-efficient cooperation in cognitive wireless powered networks". IEEE Communications Letters, $21(1)$, https://doi.org/10.1109/LCOMM.2016.2613537, 2017.

[10] B. Khal, B. Hamdaoui, M. Ghorbel, M. M. Guizani, \& X. Zhang, Joint Data and Power Transfer Optimization for Energy Harvesting Wireless Networks". IEEE INFOCOM International Workshop on Mobility Management in the Networks of the Future World Joint, 864-869. https://doi.org/10.1109/INFCOMW.2016.7562175, 2016.

[11] H.J. Zhang, "Throughput Maximization in Wireless Powered Communication Networks". IEEE Trans. Wireless Commun , pp 418-28, 2014

[12] Z.I.N Hadzi-Velkov, "Wireless networks with energy harvesting and power transfer: Joint power and time allocation". IEEE Signal Process, pp. 50-54, 2016. 